BMI, tỷ lệ VB/VM đều tăng ở cả hai giới nhóm gan nhiễm mõ̃ so với nhóm chứng.

- ĐDLMDD với ĐDLMTPM $(r=0,23 ; p<0,05)$ và ĐDLMDD với ĐDLMNT $(r=0,18 ; \mathrm{p}<0,05)$ tương quan ở mức thấp; ĐDLMNT với ĐDLMTPM $(r=0,42 ; p<0,05)$ tương quan ở mức trung bình.

- Tương quan thuận ở mức trung bình giữa ĐDLMNT với các chỉ tiêu cân nặng $(r=0,48)$, vòng bụng $(r=0,47)$ và chỉ số $B M I(r=0,42)$ với $p<0,05$; tương quan thuận ở mức thấp giữa ĐDLMDD, ĐDLMTPM với chiêu cao, cân nặng vòng bụng và chỉ số $B M I$, với $p>0,05$.

\section{TÀI LIÊUU THAM KHẢO}

1. Lê Văn Bàng (2004), "Tình hình béo phì ở đối tượng trên 15 tuổi tại thành phố Huế - Viêtt Nam", Kỷ yểu toàn văn các đề tài nghiên cứu khoa học Hội nghị Nội tiết \& Đái tháo đường toàn quốc lần thứ II, tr.666-674.

2. Suzuki R, Watanabe S, Hirai Y, et al (1993).
Abdominal wall fat index, estimated by ultrasonography, for assessment of the ratio of visceral fat to subcutaneous fat in the abdomen. Am J Med, 309-314.

3. Bô̂ Y tế: Các giá trị sinh học người Việt Nam bình thường thâap kỷ 90- Thế kỷ XX.. Nhà xuất bản $Y$ học Hà Nội, 2003.

4. Karacas P. P., Bozlir M. G. (2012), "Anthropometric indices in relation to overweight and obesity among Turkish medical students", Arch Med Sci, 8 (2), pp. 209-213

5. Silha J. V., Krsek M., Skrha J. V., et al (2003), "Plasma resistin, adiponectin and leptin level in lean and obese subjects: correlations with in insulin resistance", European Journal of Endocrinology, 149, pp. 331-335.

6. Nuran Sabir, Yurdaer Sermez, Selcuk Kazil, Mehmet Zencir (2001), "Correlation of abdominal fat accumulation and liver steatosis: importance of ultrasonographic and anthropometric measurements", European Journal of Ultrasound, pp 121-128.

7. Van Steenbergen W, Lanckmans S (1995), Liver disturbances in obesity and diabetes mellitus. Int J Obes, pp 27-36.

\title{
ĐÁNH GIÁ HIÊUU QUẢ KỸ THUÂT TIÊM NGOÀI MÀNG CỨNG DƯớI HƯớNG DẪN CẮT LỚP VI TÍNH Ở BÊNH NHÂN ĐAU DO THOÁT VỊ ĐĨA ĐỆM CộT SỐNG THẮT LƯNG
}

\author{
Phùng Anh Tuấn*, Nguyễn Xuân Khái*, \\ Dương Công Tuấn*, Nguyễn Đức Thuận*, Nguyễn Việt Dũng* \\ mức độ đau, mức độ mất chức năng sinh hoạt.

\section{SUMMARY} \\ THE EFFECTIVENESS OF CT-GUIDED \\ EPIDURAL STEROID INJECTIONS FOR LOW \\ BACK PAIN DUE TO LUMBAR DISC \\ HERNIATION
}

TÓM TẮT

Mục đích: Đánh giá hiệu quả của kỹ thuật tiêm ngoài màng cứng (NMC)dưới hướng dẫn cắt lớp vi tính (CVLT) trong điêu trị đau thắt lưng do thoát vị đĩa đệm (TVĐĐ). Đối tượng và phương pháp: 36bênh nhân (BN)đau thắt lưng do TVĐĐ được tiến hành tiêm NMC dưới hướng dẫn CVLT tại khoa $X$ quang can thiêp - Bệnh viện Quân y 103, từ 10/2019 đến tháng 09/2020. Đánh giá hiệu quả kỹ thuâtt bằng thang điểm đau VAS (visual analog scales) và thang điểm mất chức năng sinh hoạt ODI (Oswestry disability index). So sánh trước và sau tiêm bằng kiểm định $\mathrm{t}$ - test và Chi bình phương test. Kết quả: Điểm VAS giảm từ $6,11 \pm 0,89$ trước tiêm xuống còn $2,47 \pm 1,13$ sau tiêm 1 tháng. Điểm ODI giảm từ 64,67 \pm 10 trước tiêm xuống còn 24,89 $\pm 15,43$ sau tiêm 1 tháng. Khác biệt có ý nghĩa thống kê. Không cótai biến, biến chứng trong và sau khi thực hiện kỹ thuật. Kết luận: Tiêm NMC dưới hướng dẫn CLVTT là kỹ thuật an toàn và hiệu quả trong điều trị đau thắt lưng do TVĐĐ.

Tư khóa: Đau thắt lưng, tiêm ngoài màng cứng,

*Bệnh viện Quân y 103.

Chịu trách nhiệm: Nguyễn Việt Dũng,

Email: dung.nguyenviet.cdha@gmail.com

Ngày nhận bài: $1 / 2 / 2021$

Ngày phản biện khoa học: 23/2/2021

Ngày duyệt bài: 25/3/2021
Objects: Evaluating the effectiveness of epidural injection with corticoid under computed tomography guidance for treatment of low back paindue to disc herniation. Subjects and methods: 36 patients with low back pain due to disc herniation were CT - guided epidural injectedat the Interventional radiology department, 103 Military Hospitalfrom October 2019 to September 2020. All patients were evaluated by using VAS and ODI scores. Comparison of VAS and DOI scores pre -and 1 month post - injection were proceeded by $\mathrm{t}$ - test and chi square - test. Results: VAS score decreased from $6.11 \pm 0.89$ points before injection to $2.47 \pm 1.13$ points 1 month post - injection. ODI decreased from $64.67 \pm 10$ points before injection to $24.89 \pm 15.43$ points 1 month post - injection. The differences were significant. There were no complications. Conclusion: CT - guided epidural spinal injectionis a safe and effective procedure for treatmentof low back pain due to disc herniation.

Keywords: Low back pain, epidural spinal injection, visual analog scale, Oswestry disability index.

\section{I. ĐẶT VẤN ĐỀ}


Đau thắt lưng do thoát vị đĩa đêm (TVĐĐ) là bệnh tương đối phổ biến trong những năm gần đầy. Mặc dù không nguy hiểm, bệnh ảnh hưởng nhiều đến công việc và sinh hoạt thường ngày của bệnh nhân (BN). Ở Việt Nam, tỷ lệ người đau thắt lưng do TVĐĐ chèn ép chiếm tới 27,75\%[1]. Bệnh thường gặp ở độ tuổi lao động nên việc điều trị có ý nghĩa kinh tếxã hội to lớn. Có nhiều phương pháp điều trị bênh đã được áp dụng bao gồm: nội khoa, vật lý trị liệu, phẫu thuật... Trên thế giới, tiêm ngoài màng cứng (NMC) dưới hướng dẫn cắt lớp vi tính (CLVT) đã được xác định là phương pháp có nhiều ưu điểm [7]. Vị trí đâu kim được kiểm soát tốt, khoang NMC được xác định chính xácđảm bảo tiêm thuốc đúng vị trí, mang lại hiệu quả cao và an toàn. Tuy nhiên ở Việt Nam, phương pháp này vẫn chưa được áp dụng nhiều. Nghiên cứu của chúng tôi được tiến hành nhằm muc tiêu đánh giá hiệu quả kỹ thuật tiêm NMC dưới hướng dẫn CLVT ở BN đau lưng do TVĐĐ cột sống thắt lưng.

\section{II. ĐỐI TƯợNG VÀ PHƯƠNG PHÁP NGHIÊN CỨU}

2.1. Đối tượng nghiên cứu. $36 \mathrm{BN}$ khám tại Phòng khám bệnh, Bệnh viện Quân y 103, được chẩn đoán xác định đau thắt lưng do TVĐĐ và được tiêm NMC dưới hướng dẫn CLVT tại khoa X Quang can thiệp từ tháng 10/2019 đến tháng $9 / 2020$.

\section{*Tiêu chuẩn chọn bênh nhân:}

- BN được chẩn đoán xác định đau thắt lưngdo TVĐĐ dựa vào khám lâm sàng kết hợp với hình ảnh cộng hưởng từ ( $\mathrm{CHT})$.

- BN có chỉ định tiêm NMC: đã điều trị bằngthuốc giảm đau, vật lí trị liệu hoặc kết hợp các phương pháp trên trong thời gian ít nhất 2 tuần nhưng không hiệu quả.

*Tiêu chuẩn loai trừ:

- BN có chống chỉ định chọc NMC: đang có nhiễm trùng vùng tiêm, bệnh lý rổi loạn đông máu...

- BN dị ứng với thuốc (thuốc cản quang, thuốctê tai chỗ, corticosteroid...).

- BN không theo dõi được kêt quả điều trị.

2.2. Phương pháp nghiên cứu. Tiến cứu, can thiệp không đối chứng, so sánh trước và sau điêu tri.

\section{Tiến hành kỹ thuật:}

- Thực hiện tại phòng chụp CLVT. Thực hiện trên máy CLVT 2 dãy, nhãn hiệu Somato Spirit, hãng SIEMENS, Đức.

- BN nằm sấp. Chụp CLVT xác định vị trí chọc kim, dự kiến hướng đi kim, độ sâu cần chọc.

- Choc kim, kiểm tra vi trí đâu kim. Tiềm $1 \mathrm{ml}$ dung dịch cản quang $10 \%(1 \mathrm{ml}$ Xenetic $+10 \mathrm{ml}$ nước cất), thấy thuốc lan trong khoang NMC.

- Tiến hành block - test bằng $1 \mathrm{ml}$ Lidocain $1 \%$, BN thây giảm đau rõ rệt

- Tiêm ngoài màng cứng 01 lọ Diprospan $7 \mathrm{mg} / \mathrm{ml}$.

Tai biến của kỹ thuật: $B N$ được theo dõi tác dụng phụ và biến chứng trong lúc làm thủ thuật, sau thủ thuật trong vòng3 giờ.

\section{Đánh giá hiệu quả kỹ thuật}

- Đánh giá hiệu quả giảm đau: So sánh điểm VAS (Visual analogue scale) tại các thời điểm trước tiêm, ngay sau tiêm và sau 1 tháng (phỏng vấn qua điện thoại).

- Đánh giá cải thiện chức năng sinh hoạt:So sánh điểm ODI(Oswestry disability index) tại các thời điểm trước tiêm và sau tiêm 1 tháng (phỏng vấn qua điện thoại).

Các thang điểm sử dụng trong nghiên cứu

- Thang điểm VAS: Đánh giá mức độ đau theo 11 điểm từ 0 tương ứng không đau đến 10 tương ứng đau không chịu nổi. Từ 1-3: đau nhẹ. 4-7: đau vừa. 8-10: đau nặng [4].

- Thang điểm ODI: Đánh giá tổng hợp mức độ ảnh hưởng sinh hoạt do đau dựa trên 10 tiêu chí: Mức độ đau, tự chăm sóc cá nhân, nâng đồ vật, đi bộ, ngồi, đứng, ngủ, hoạt động tình dục, hoạt động xã hội, đi du lịch. Điểm ODI 0: hoàn toàn bình thường và 100 mất hoàn toàn chức năng. Căn cứ vào điểm $\mathrm{ODI}$, tình trạng mất chức năng được chia làm 5 mức độ. Độ 1 (mất chức năng nhẹ): ODI $0-20$. Độ 2 (mất chức năng vừa): ODI 21-40. Độ 3 (mất chức năng nặng): ODI 41-60. Độ 4 (mất chức năng rất nă̆ng): ODI 61-80. Độ 5 (mất hoàn toàn chức năng): ODI 81-100 [4].

Phân tích và xử lý số liệu

- Sử dụng phần mềm thống kê SPSS 22.0.

- So sánh điểm VAS và ODI trước và sau can thiệp bằng thống kê $\mathrm{t}$ - test, so sánh tỷ lệ bằng thổng kê Chi bình phương test.

\section{KẾT QUẢ NGHIÊN CứU}

3.1. Đặc điểm chung của nhóm nghiên cứu

- 36 BN gồm10 nam và 26 nữ, tỷ lệ nữ/nam là $2,6 / 1$.

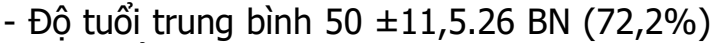
trong độ tuổi $40-60$.

- Phẩn bố vị trí thoát vị: L3/4: 1 BN $(2,8 \%)$, L4/5: 10 BN $(27,8 \%)$, L5/S1: 5 BN $(13,9 \%)$, $L 3 / 4$ và $L 4 / 5: 3 B N(8,3 \%), L 4 / 5$ và $L 5 / S 1: 8 B N$ $(22,2 \%)$, tổn thương >2 tầng: 9 BN (25\%).

- Tất cả BN đều tiêm 1 lần.

\subsection{Hiệu quả điều trị}


Bảng 1. Thay đổi điểm VAS và điểm $O D I$ trước và sau tiêm ngoài màng cứng $(n=36)$

\begin{tabular}{|c|c|c|c|c|}
\hline $\begin{array}{c}\text { Thang } \\
\text { điểm }\end{array}$ & $\begin{array}{c}\text { Trước } \\
\text { tiêm }\end{array}$ & $\begin{array}{c}\text { Ngay } \\
\text { sau } \\
\text { tiêm }\end{array}$ & $\begin{array}{c}\text { Sau } \\
\text { tiêm } \\
\text { 1tháng }\end{array}$ & p \\
\hline VAS & $\begin{array}{c}6,11 \pm \\
0,89\end{array}$ & $\begin{array}{c}2,11 \pm \\
0,89\end{array}$ & $\begin{array}{c}2,47 \pm \\
1,13\end{array}$ & $\begin{array}{c}< \\
0,05\end{array}$ \\
\hline ODI & $\begin{array}{c}64,67 \pm \\
10,00\end{array}$ & - & $\begin{array}{c}24,89 \pm \\
15,43\end{array}$ & $<$ \\
& & 0,05 \\
\hline
\end{tabular}

$\mathrm{P}: \mathrm{t}$ - test

Nhận xét: Điểm VAS và điểm ODI sau tiêm 1 tháng đều giảm có ý nghĩa so với trướctiêm. Tuy nhiên điểm VAS sau tiêm 1 tháng cao hơn có ý nghĩa so với điểm VAS ngay sau tiêm

Bảng 2. Thay đổi mức độ đau và mất chức năng sinh hoạt $(n=36)$

\begin{tabular}{|c|c|c|c|c|}
\hline \multicolumn{2}{|c|}{ Đặc điểm } & \multicolumn{2}{|c|}{\begin{tabular}{c|c} 
Trước & Sau tiêm \\
tiêm & 1 tháng \\
\end{tabular}} & \multirow{2}{*}{$\mathbf{p}$} \\
\hline \multirow{3}{*}{$\begin{array}{l}\text { Mức độ } \\
\text { đau }\end{array}$} & Nhẹ & $0(0)$ & $28(77,8)$ & \\
\hline & Vừa & $27(75)$ & $8(22,2)$ & \multirow[t]{2}{*}{$<0,05$} \\
\hline & Nặng & $9(25)$ & $0(0)$ & \\
\hline \multirow{4}{*}{\begin{tabular}{|c|} 
Mức đố \\
mất chức \\
năng sinh \\
hoạt
\end{tabular}} & Nhe & $0(0)$ & $19(52,8)$ & \multirow{4}{*}{$<0,05$} \\
\hline & Vừa & $0(0)$ & $10(27,8)$ & \\
\hline & Nặng & $10(27,8)$ & $7(19,4)$ & \\
\hline & Rất nặng & $26(72,2)$ & $0(0)$ & \\
\hline
\end{tabular}

P: Chi bình phương test.

Nhận xét: Sau tiêm 1 tháng, mức độ đau cũng như mức độ mất chức năng sinh hoạt giảm so với trước tiêm, $\mathrm{p}<0,05$.

Tai biến, biến chứng. Trong quá trình thực hiện thủ thuật, chúng tôi không gặp bất kỳ trường hợp nào xảy ra tai biến, biến chứng trong và sau quá trình tiêm.

\section{BÀN LUẬN}

Trong nghiên cứu của chúng tôi, độ tuổi trung bình của các BN $55 \pm 11,5$, tương đương với kết quả nghiên cứu Vi Thị Hải $55 \pm 10,04$ [3]. Về độ tuổi mắc bệnh, nhóm tuổi hay gặp nhất là từ $40-60$ tuổi (chiếm 72,2\%). Lứa tuổi này hay gặp do quá trình thoái hóa sinh học của đĩa đệm cũng như tác động cơ học của chấn thương, vi chấn thương trong quá trình lao động. Kết quả nghiên cứu của chúng tôi cho thấy nữ giới chiếm tỷ lệ cao hơn $(72,2 \%)$, tỷ lệ nữ/nam 2,6/1. Nguyên nhân có thể do nữ giới đang dần dần làm những công việc nặng như nam giới, đồng thời do trải qua thời kì mang thai và nuôi con, $B N$ nữ thường chỉ nhập viện khi bệnh đã ảnh hưởng nhiều đến sinh hoạt hàng ngày. Độ tuổi 40-50 tuổi là độ tuổi mãn kinh ở phụ nữ, nhiều yếu tố rối loan nội tiết gây loãng xường, thoái hóa đĩa đệm dể dẫn đến TVĐĐ.

Trong nghiên cứu của chúng tôi, tổn thương hay gặp nhất ở tầng đĩa đệm L4/5, L5/S1 và kết hợp cả 2 đĩa đệm này (23/36 BN, 63,9\%). Nhóm BN có tổn thương $>2$ tầng đĩa đệm chiếm $25 \%$. Kết quả này phù hợp với các nghiên cứu khác. Trong nghiên cứu của Lê Hà Năng Chưởng, 70\% $B N$ có tổn thương đĩa đêm $L 4 / 5,33,3 \%$ BN có tổn thương đĩa đệm L5/S1 [2]. Tầng đĩa đệm $L 4 / 5$ và $L 5 / S 1$ là vùng bản lề của cột sống, có biên độ vận động lớn, chịu trọng tải lớn của cả cơ thể, do đó dễ bị thoát vị nhất.

Trong nghiên cứu của chúng tôi, tất cả các BN đều được tiêm NMC dưới hướng dẫn của CLVT. Hình ảnh CLVT giúp người thực hiện nhìn rõ các cấu trúc giải phấu và đảm bảo việc chọc kim và đưa thuốc vào đúng vị trí. Vì vậy, sử dụng CLVT hướng dẫn tiêm NMC đã được đưa vào các khuyến cáo trên thế giới. Trong nghiên cứu của Botwin KP, chọc NMC không có chẩn đoán hình ảnh (CĐHA) dẫn đường có thể sai vị trí từ $25-40 \%$ [5]. Nghiên cứu của Kuouki S chia $40 \mathrm{BN}$ làm 2 nhóm tiêm có hướng dẫn CLVT và không. Kết quả cho thấy tỉ lệ giảm điểm ODI sau 30 ngày ở nhóm có hướng dẩn CLVT là $40 \%$ so với $10 \%$ của nhóm tiêm không có hướng dẫn. Tất cả các $B N$ của chúng tôi đều chỉ tiêm 1 lần bằng Diprospan $7 \mathrm{mg} / \mathrm{ml}$. Khi tiến hành tiêm dưới hướng dẫn $\mathrm{C} Đ H A$, vị trí tiêm có thể xác định được chính xác, do đó, việc lặp lại lần thứ hai là khổng cần thiết. Kim $B R$ cho rẳng việc tiêm 1 mũi và theo dõi đánh giá có hiệu quả tốt hơn so với việc tiêm lặp lại mũi thứ hai trong vòng 3 tuần đầu tiên sau mũi thứ nhất.

Đau là biểu hiện dễ thấy nhất của TVĐĐ cột sống thắt lưng và cũng là nguyên nhân chính khiến $B N$ phải điều trị. Đau cũng chính là yếu tố chính gây hạn chế vận động, sinh hoạt, ảnh hưởng đến chất lượng cuộc sống của BN. Trong nghiên cứu này, chúng tổi nhận thây có sự cải thiện rõ rệt về mức độ đau giứa thời điểm trước và sau tiểm. Điểm VAS giảm tới 4 điểm ở thời điểm ngay sau tiêm. Sự khác biệt này là có ý nghĩa thống kê, $p<0,05$. Thời điểm này, tác dụng giảm đau chủ yếu do tác dụng gây tê tại chỗ của Lidocain khi làm block - test. Sau đó tác dụng kháng viêm của Diprospan giúp giảm tình trạng đau của BN. Sau tiêm 1 tháng, điểm VAS tăng có ý nghĩa thống kê so với thời điểm ngay sau tiêm mă̆c dù vẫn giảm hơn so với trước tiêm. Điều này được giải thích do $\mathrm{BN}$ trong nghiên cứu thường là lao động chính trong gia đình. Khi triệu chứng đau giảm, họ lại tiếp tục lao động mà không chú ý các biện pháp để tránh gây áp lực lên cột sống khiến cho đau lại tăng trở lại. Tất cả những $\mathrm{BN}$ này đều được khuyến cáo nển hạn chế lao động nặng, sử dụng nẹp lưng để hỗ 
trợ cột sống.Nghiên cứu của Lê Năng Hà Chưởng trên $30 \mathrm{BN}$ TVĐĐ được tiêm NMC dưới hướng dẫn CLVT cho thấy điểm VAS giảm 5,5 điểm sau tiêm 1 ngày, giảm 5,8 điểm sau tiêm 3 tháng, sự khác biệt có ý nghĩa thống kê [2]. Gruenberg nghiên cứu trên $38 \mathrm{BN}$ nhận thấy điểm đau giảm từ $7,6 \pm 2,2$ điểm xuống còn $2,3 \pm 2,6$ điểm sau 1 tháng điều trị [7].

Đánh giá theo mức độ đau, chúng tôi nhận thấy trước tiêm tất cả $36 \mathrm{BN}$ đều đau vừa và nặng. Sau tiêm 1 tháng có sự giảm mạnh mức độ đau với đa số $\mathrm{BN}$ đau nhe $(77,8 \%)$ và không có $B N$ nào đau nặng. Sự khác biệt về mức độ đau trước và sau tiêm là có ý nghĩa thống kê, $p<0,05$. Trong nghiên cứu của Lê Hà Năng Chưởng, $100 \% \mathrm{BN}$ trước tiêm ở mức đau vừa và nặng. Sau tiêm, nhóm đau vừa và nặng giảm còn $36,7 \%$ [2]. Kết quả này tương đương với nghiên cứu của chúng tôi.

Thang điểm ODI được sử dụng để đánh giá tổng hợp mức độ ảnh hưởng sinh hoạt do đau của BN. Thang điểm gồm 10 tiêu chí và đước tính điểm từ 0 hoàn toàn bình thường đến 100 mất hoàn toàn chức năng sinh hoạt. Trong nghiên cứu của chúng tôi, sau tiêm 1 tháng, điểm ODI giảm từ 39,78 $\pm 13,86$ điểm, xuống còn $24,89 \pm 15,43$ điểm. Mức giảm này là có ý nghĩa thống kê, $p<0,05$. Trước tiêm, $100 \% \mathrm{BN}$ mất chức năngsinh hoạt nặng và rất nặng, trong đó chủ yếu ở mức rất nặng $(72,2 \%)$. Sau tiêm 1 tháng, chỉ còn $19,4 \%$ BN mất chức năng sinh hoạt ở mức nặng, còn lại đa số BN mất chức năng sinh hoạt ở mức nhẹ và vừa. Như vậy, tiêm NMC dưới hướng dẫn CLVT có hiệu quả rất tốt trong việc cải thiện chức năng sinh hoạt ở BN TVĐĐ. Trong nghiên cứu của ArdenNK có $60 \mathrm{BN}$ (chiếm $50 \%$ số BN nghiên cứu)giảm $75 \%$ điểm ODI [4]. Theo Lê Năng Hà Chưởng, sau 3 tháng điểm ODI giảm 28,8 điểm. Nhóm có điểm ODI ở mức nặng và rất nă̆ng giảm từ $93,3 \%$ xuống còn $20,0 \%[2]$. Các kết quả này tương đương với nghiên cứu của chúng tôi.

Trong nghiên cứu của chúng tôi, tất cả $36 \mathrm{BN}$ tiêm NMC dưới hướng dẫn CLVT không gặp bất kỳ tai biến biến chứng nào trong và sau tiêm. Tiêm NMC là một trong những phương pháp hiệu quả và an toàn trong điều trị bệnh lý đau rế thẩn kinh do TVĐĐ, tuy nhiên nểu tiến hành phương pháp không đúng chỉ định hoặc không tuân thủ các quy trình tiêm, có thể gặp một vài tác dụng phụ không mong muốn. Chọc thủng màng cứng dẫn đến hạ huyết áp nội sọ, tụ máu dưới màng cứng. Ngoài ra có thể gặp chấn thương tủy sống hoặc dây chằng, liệt một bên do tiêm thuốc vào động mạch đốt sống [6]. Vi Thi Hải nhận thấy trong số 60 BN được tiêm NMC ở cả hai nhóm, không có BN nào có tai biến, biến chứng. $2 \mathrm{BN}(6,7 \%)$ tiêm NMC qua khe cùng cụt đau tăng lên và tự khỏi sau 3 ngày [3]. Hình ảnh CLVT giúp xác định chính xác đâu mũi kim và hạn chế những tai biến biến chứng có thể xảy ra.

\section{KẾT LUÂNN}

- Điểm VAS trước tiêm $6,11 \pm 0,89$ giảm có ý nghĩa còn $2,11 \pm 0,89$ ngay sau tiêm và $2,47 \pm$ 1,13 sau tiêm 1 tháng.

- Điểm ODI trước tiêm $64,67 \pm 10$ giảm có ý nghĩa còn $24,89 \pm 15,43$ sau tiêm 1 tháng.

- Mức đô đau và mức đô mất chức năng sinh hoạt giảm có ý nghĩa sau tiểm.

- Tiêm ngoài màng cứng dưới hướng dẫn cắt lớp vi tính là phương pháp an toàn không có tai biến, biến chứng.

\section{TÀI LIỆU THAM KHẢO}

1. Nguyen Van Chuong, Dinh Cong Pho, Nguyen Thi Thanh Thuy et al. (2019). Pain incidence, assessment, and management in Vietnam: a crosssectional study of 12,136 respondents. Journal of Pain Research, 12, 769-777.

2. Lê Năng Hà Chưởng (2019). Nghiên cứu hiệu quả giảm đau của kĩ thuật tiêm thẩm phân ngoài màng cứng vùng thắt lưng dưới hướng dẫn cắt lớp vi tính. Luận văn Cao học, Đại học Y Hà Nôi.

3. Vi Thi Hải (2014). Đánh giá hiệu quả tiểm ngoài màng cứng qua khe liên đốt L4 - L5 băng hydrocortisone acetat trong điều trị đau dây thần kinh tọa do thoát vị đĩa đệm. Luận văn Cao học, Đại học Y Hà Nội.

4. Arden NK, Price $C$, Reading I, et al. (2005). A multicentre randomized controlled trial of epidural corticosteroid injections for sciatica: the WEST study. Rheumatology (Oxford), 44(11), 1399-1406.

5. Botwin KP, Gruber RD, Bouchlas CG,et al. (2002). Fluoroscopically guided lumbar transformational epidural steroid injections in degenerative lumbar stenosis: an outcome study. Am J Phys Med Rehabil, 81(12), 898-905.

6. Epstein NE (2017). Neurological complications of lumbar and cervical dural punctures with a focus on epidural injections.Surg Neurol Int,8(1),60.

7. Gruenberg MF, Petracchi $M$, Valacco $M$, et al. (2011). Use of CT-guided periradicular injection for the treatment of foraminal and extraforaminal disc herniations. Evid Based Spine Care J, 2(3), 19-24.

8. Jeong $\mathrm{YC}_{\text {, Lee }} \mathrm{CH}$, Kang S,et al. (2017). Contrast spread in the superoposterior approach of transforaminal epidural steroid injections for lumbosacral radiculopathy. Ann Rehabil Med, $41(3), 413-420$. 